\title{
CATALOGO DE FORMULARIOS NOTARIALES DEL ARCHIVO PARROQUIAL DE AYORA
}

\author{
$M^{a}$ MILAGROS CARCEL ORTI \\ Dpto. Paleografía y Diplomática \\ UNIVERSIDAD DE VALENCIA
}

\section{INTRODUCCION}

Recientemente ha visto la luz impresa un trabajo donde se dan a conocer, a modo de inventario, los fondos manuscritos del Archivo Parroquial de Ayora (1). En la Sección XXII, que el autor titula Protocolos y minutarios notariales y concretamente en el número 30 , se señalan tres formularios notariales que hemos tenido ocasión de consultar, cuya ficha catalográfica y rúbricas de los instrumentos registrados damos a continuación.

El primer formulario, con fechas extremas desde 1570 a 1591, se encuentra en el protocolo de Juan Martínez. Consta de un total de 209 rúbricas en latín, correspondientes a otros tantos documentos copiados, bien en latín o castellano, como es habitual en este tipo de formularios, es decir, sustituyendo los nombres propios o las cantidades por la partícula talis o por una simple T. Las rúbricas están ordenadas alfabéticamente al comienzo del libro, remitiendo al folio donde se encuentra desarrollada cada una de ellas. Hay que destacar como nota curiosa la aposición en algunas de la frase in lingua betica para indicar

(1) Cfr. PONS ALOS, V., El Archivo Parroquial de Ayora: fondos manuscritos, "Anales de la Universidad de Alicante. Historia Medieva/w, 2 (1983), 273-319. 
que el documento a que se refiere está redactado en castellano (2).

A continuación del índice de rúbricas lleva anotada una receta para hacer tinta cuyo contenido daremos a conocer en un próximo trabajo. Sigue luego un juramento de notarios y unas Diffinitiones et regule ad tabelliones decoradum valde necessarie. Por último, aparecen las fórmulas con las rúbricas en el centro de la hoja y glosas en los márgenes.

El segundo formulario — de 1587- está incompleto faltándole las 32 primeras hojas, donde seguramente iría el índice de rúbricas. Consta de 25 documentos copiados con las correspondientes abreviaturas Ilevando al final cosido a los cuadernillos y redactado in extensu el testamento de Catalina Cinca, vecina de Alcañiz, realizado ante Cristóbal de Herrera, notario público de dicha villa. El resto de documentos hacen alusión a personas de la ciudad de Valencia, al igual que los del formulario anterior.

Este formulario se atañe a Alonso Muñoz, notario público de Ayora (3), debido a la frecuencia de documentos expedidos por el mismo, afirmación que tendrá que confirmarla un análisis comparativo de su escritura.

El formulario de 1672, también incompleto, lleva en la parte posterior una Tabla de las articulatas que ai en esse formulario, es decir, un índice de 71 rúbricas, dispuestas en el mismo orden que figuran en el formulario. Al final del índice hay una nota de la misma mano y color de tinta que éste donde se lee Pedro Pardo, 44 años, pudiendo ser el autor del formulario. De los documentos se copian solamente las cláusulas que interesan, llevando a continuación y subrayadas una explicación de las mismas en castellano. Los datos que en ellas se leen aluden a la ciudad de Valencia.

\section{FICHA CATALOGRAFICA}

$X X I I-30.1$

[FORMULARIO DE JUAN MARTÍNEZ]. 1570, junio, 13-1591.

438 hojas. $220 \times 160 \mathrm{~mm}$. Encuadernación en pergamino, falta la tapa delantera, presenta refuerzos de cuero en en el lomo, cuadernos cosidos.

Humanística corriente y cursiva. Latín y castellano.

Afectado por manchas de humedad y de óxido férrico.

(2) Corresponde a las rúbricas 22, 56, 76, 157, 158, 166 y 167.

(3) Crr. PONS ALOS, V., Op. cit., p. 318. 
[FORMULARIO DE ¿ALONSO MUÑOZ?]. 1587, ENERO, 10.

67 hojas, numeradas de la 33 a la $48.220 \times 160 \mathrm{~mm}$. Cuadernos cosidos por el lomo, sin cubiertas, incompleto.

Humanística corriente y cursiva. Latín y castellano.

Afectado por manchas de humedad y de óxido férrico.

XXII-30.3

[FORMULARIO DE ¿PEDRO PARDO?]. 1672, mayo, 4.

77 hojas, numeradas de la 16 a la $88.220 \times 160 \mathrm{~mm}$. Cuadernos cosidos por el lomo con refuerzos de papel en el mismo. Incompleto.

Humanística corriente. Catalán.

Buen estado de conservación.

APENDICE DE RUBRICAS

1

FORMULARIO DE JUAN MARTÍNEZ

INDEX INSTRUMENTORUM INMEDIATE SEQUENTIUM

A

[1] Aumentum sive afigmentum dotis (f. 115)

[2] Asecuramenteum dotis et anuenti (f. 161)

[3] Acteptacio de resperit racione cuiusdam arendamenti exhimendo quandam fidanciam et dando aliam (f. 119)

[4] Adopcio filii alieni (f. 169)

[5] Afirmamentum pueri ad adepiscendum officium (f. 165)

[6] Apoca racione vendicionis ( $f$. 171)

[7] Asignacio specialis obligacio et nova consignacio (f. 2)

[8] Aprobacio cuiusdam bendicionis per dominium directum (f. 34)

[9] Afirmamenteum puelle ad serviendum ( $f$. 38)

[10] Arrendamentum domorum (f. 40)

[11] Almoneta facta per tutorem et curatorem (f. 59)

[12] Abolicio denunciacionis lacioris vulneris ( $f$. 18)

[13] Apoca dotis facta (f. 218)

[14] Apoca dicti precii racione vendicionis (f. 111)

[15] Apoca racionis cancellacionis debiti (f. 224)

[16] Apoca carricamenti censualis prout instrumentum (f. 19)

[17] Addicio inventarii post inventarium confectum (f. 148)

[18] Apoca racione pensionis censualis (f. 202)

[19] Afirmamentum ad adipiscendum officium (f. 90)

[20] Abolicio sive perdo valde dificilis (f. 53)

[21] Apocad dotis (f. 179)

[22] Abolicio sive perdo in lingua betica (f. 230)

[23] Apoca per viam diffinitionis (f. 225) 
[24] Apoca et carta de costo (f. 231)

[25] Abolicio sibe perdo (f. 251)

C

[26] Carricamentum censualis cum pacto de succedendo in iuribus prioritatis et pocioritatis (f. 114)

[27] Carricamentum censualis ad opus luhendi et quitandi aliud censum cum pacto de succedendo (f. 128)

[28] Carricamentum censualis a universitate factum prout in prothocollo (f. 10)

[29] Carricamentum censualis cum eius apoca prout instrumenta (f. 1)

[30] Carricamentum violari prout in prothocollo (f. 15)

[31] Consignacio pensionum censualis cum eius instrumentum (f. 17)

[32] Cessio pro luendis in tot vicibus aliquo census (f. 129)

[33] Cessio ante pactu (f. 102)

[34] Cessio pro solvendis (f. 14)

[35] Capitulacio prout in prothocolio (f. 32)

[36] Codicillum cum eius publicacione (f. 45)

[37] Comendacio de certa quantitate ( $\uparrow$. 58)

[38] Constitucio principalis obligacionis in aliquo censum (f. 66)

[39] Constitucio principalis obligacionis in certa quantitate (f. 67)

[40] Caputbrevium quarumdam pensionum (f. 118)

[41] Caputbrevium domorum et terrarum (f. 244)

[42] Constitucio baiuli sue creacio (f. 120)

[43] Compromissum cum eius intimas et sentenciis (f. 134)

[44] Comanda cadaveris sive de cos mort (f. 164)

[45] Comendacio bonorum mobilium (f. 223)

[46] Capitulacio inter partes prout in prothocollo (f. 234)

[47] Carricamentum violarii prout in prothocollo (f. 149)

[48] Conssignacio pensionum diti violarii (f. 152)

[49] Constitucio principalis obligacionis (f. 92)

[50] Carricamentum censualis prout in prothocollo (f. 237)

[51] Clausula minus falimenti (f. 246)

[52] Concessio moratorie et iudicies quinquenales (f. 141)

[53] Comendacio testamentum ( $f$. 143)

[54] Carricamentum censualis en pla cum apoca et promissione (f. 32)

[55] Cancellaçio censualis (f. 52)

[56] Cessio facta in lingua betica (f. 52)

[57] Capbreu (f. 54)

[58] Donacio prout in forma (f. 183)

[59] Delegacio ad capbrevandum (f. 117)

[60] De relinquimentum quarumdam terrarum (f. 121)

[61] Debitum cum responsione interesse racione vendicionis ( $f$. 163)

[62] Debitorium cum eius cancellacione (f. 151)

[63] Diffinicio prout in prothocollo (f. 166) 
[64] Donacio generalis cum eius insinuacione (f. 172)

[65] Donacio ad sacras ordines acipiiendas (f. 174)

[66] Donacio seu patrimonium ad sacras ordines accipiendas (f. 186)

[67] Donacio facta in contemplacione matrimonii (f. 214)

[68] Debitum simplex (f. 213)

[69] Diffinicio per moderni apoca (f. 223)

[70] Diffinicio per modum apoce (f. 239)

[71] Debitorium cum responsione interesse ex precio cuiusdam vendicionis cum de cara et aliena villa certarum terrarum (f. 153)

[72] Delegacio advocati cum constitucione salarii in duabus solucionibus (f. 201)

[73] Depositio simplex (f. 245)

[74] Donacio generalis cum eius insinuacione (f. 44)

[75] Debitorium sive obligacionum com a comprador de cort [...] acabada (f. 140)

[76] Debitorium sive obligacio in lingua betica (f. 243)

[77] Diffinicio et absolucio (f. 140)

[78] Donacio facte ad sacras ordines percipiendas (f. 140)

E

[79] Exempcio cuiusdam specialis obligacionis (f. 20)

[80] Electio iudicis in causis inphiteoticalibus ( $f$. 55)

[81] Exempcio cuiusdam obligacionis (f. 167)

[82] Exempcio cuiusdam arrendamenti (f. 220)

[83] Elongacio procuracionis ad vendendum censuale (f. 151)

[84] Elongacio procuracionis ad cessionem conficiendam (f. 145)

[85] Elongacio compromissum (f. 137)

[86] Elongacio ad firmandum quitamentum de certa quantitate ( $f$. 138)

F

[87] Firma domini directi in aliquo vendicione (f. 54)

[88] Franquitatis servum prout in instrumenta ( $f$. 187)

[89] Inventarium factum per tutorem et curatorem (f. 61)

[90] Inventarium factum a herede (f. 219)

[91] Indempnitatis racione carricamenti censualis (f. 19)

[92] Indempnitatis raçione cambii (f. 176)

[93] Insinuacio donaçionis (f. 128)

[94] Indempnitas racione cuiusdam obligacionis (f. 80)

[95] Laudacio et aprobacio per dominum directum racione carricamenti censualis (f. 22)

[96] Licencia a dominio directo ad carricandum super quadam domo (f. 29) 
[97] Licencia per dominum directum ad edifficandam domum (f. 30)

[98] Laudacio cuiusdam vendicionis facta per uxorem a marito vendita (f. 127)

[99] Liçençia ad carricandum per dominum directum (f. 246)

[100] Laudacio et aprobacio cuiusdam cessionis (f. 173)

N

[101] Nubcialia in qua mulier se dotat (f. 36)

[102] Nubcilia secundum forum Valencie cum qua pater dotat filia (f. 105)

[103] Nubcialia partim in pecunia partim in canpis et in ortalibus (f. 159)

[104] Nubcialia societatis sive germania ( $f$. 160)

[105] Nubcialia in qua maritus dotat uxoris (f. 215)

[106] Nubcialia iurata (f. 217)

[107] Nubcialia societatis sive germania (f. 247)

[108] Nubcialia sive constitucio dotis habite et nun dum inscriptis redacte (f. 112)

[109] Nubcialia a dot [...] cum germania (f. 139)

[110] Nubcialia factum per viduam [...] et germania enarativa (f. 179)

[111] Nubcialia societatis et germanie deducta capitalia (f. 232)

o

[112] Obligacionum responsione interesse (f. 231)

$\mathbf{P}$

[113] Procuracio ratifficando et conssumando (f. 47)

[114] Procuracio generalis et ad habendum (f. 48)

[115] Procuracio ad presentandum aliquam beneficiatum in aliquo benefficio (f. 50 )

[116] Procuracio ad instandum aliquod testamentum ut publicetur (f. 74)

[117] Procuracio ad tradendam alicui possessionem de quadam loco racione vendicionis (f. 84 )

[118] Procuracio ad fermandum et contra fermandum de iure (f. 99)

[119] Procuracio cum constitucione salarii (f. 104)

[120] Procuracio ad compromittendum (f. 108)

[121] Procuracio ad putandos intimas extraiudiciales (f. 113)

[122] Procuracio ad comparendum coram iudice dellegato (f. 113)

[123] Procuracio ad extrahendum pecunias a tabula seu banquis (f. 114)

[124] Procuracio ad deshaveynandum (f. 122)

[125] Procuracio ad comparendum coram $T$ et renunciandum baiuliani et alcaydiatum ville de $T$ in posse filii (f. 123)

[126] Procuracio ad acceptandum possessionem alicui beneficii (f. 124)

[127] Procuracio ad derelinquendum aliquas domos in posse juratorum alicuivus ville (f. 125) 
[128] Procuracio ad acceptandum legata (f. 126)

[129] Procuracio ad presentandum instrumentum franquitatis ab aliquo (f. 130)

[130] Procuracio ad desponsandum cum designata muliere (f. 131)

[131] Procuracio ad nominandum gubernatorem (f. 132)

[132] Procuracio ad dotandum filium in aliquo matrimonio (f. 132)

[133] Procuracio ad vendendum aliquas domos cum terras (f. 240)

[134] Procuracio ad afirmandum aliquam puellam (f. 157)

[135] Procuracio ad littes tantum prout in prothocollo (f. 200)

[136] Procuracio ad littes ad longum (f. 204)

[137] Procuracio ad renunciandum gubernatorem (f. 199)

[138] Procuracio ad ostendendum franqueam ubicuius ( $f$. 226)

[139] Procuracio cancellandum instrumentum obligacionis (f. 198)

[140] Procuracio ad littes et ad cabrevandum domos, terras et alias res (f. 203)

[141] Possessio domorum prout in forma (f. 63)

[142] Possessio cuiusdam loci tradita per procuratorem et accepta virtute cuiusdam vendicionis ( $f$. 85)

[143] Possessio cuiusdam valle sive baronia accepta per procuratorem prout in forma (f. 88)

[144] Possessio beneficii (f. 178)

[145] Possessio cuiusdam domus sive canongia valde difficile (f. 180)

[146] Provissio quitandi aliquod censum (f. 210)

[147] Provissio de dando aliam specialem obligacionem (f. 18)

[148] Permutacio instrumentorum (f. 21)

[149] Particio seu divisio inter fratres (f. 69)

[150] Particio inter heredes de nonnulis bonis per socium electionem (f. 72)

[151] Prorrogacio compromissi ad composicionem T dictis (f. 76)

[152] Permutacio compromissi ad composicionem (f. 77)

[153] Particio facta inter maritum et uxorem racione societatis sive germania morte perdurante (f. 106)

[154] Pacamentum dotis per se ipsam factum seu insolutum dacionis causa mortis viri sui (f. 109)

[155] Pacamentum dotis et aumenti (f. 221)

[156] Procuracio ad vendendum domos, terras (f. 240)

[157] Procuracio ad habendum in lingua betica ( $f$. 242)

[158] Pax in lingua betica (f. 236)

[159] Procuracio generalis tantum (f. 207)

[160] Pacamentum cum capitalis racione dotis (f. 152)

[161] Procuracio ad cancellandum aliquod censualis (f. 228)

[162] Procuracio ad firmandum quitamentum de aliquo census (f. 229)

[163] Instrumentum pro is ratifficando et confirmando (f. 47)

[164] Possessio de aliquibus bonis ad sacras ordines percipiendas (f. 140)

[165] Instrumentum pro is ad cancellandum debitorium (f. 248)

[166] Instrumentum pro is in lingua betica ad habendum 200 arum regaliorum beticorum ( $f .55$ )

[167] Instrumentum pro is generalis in lingua betica (f. 55)

[168] Procuracio censualis ( $f$. 249)

[169] Procuracio ad habendum et vendendum (f. 250)

[170] Procuracio ad presentandum beneficium (f. 253) 
[171] Quitamentum censualis prout in forma (f. 51)

[172] Quitamentum partis censualis (f. 78)

[173] Quitamentum prout in prothocollo (f. 227)

[174] Quod non curat pensio censualis quia instrumenta no fuerunt exhibito a domino censualis (f. 75)

R

[175] Restitucio dotis et aumenti per maritum uxori (f. 24)

[176] Restitucio iurium senali qua cessione (f. 31)

[177] Revocacio procuratoris cum eius interposita (f. 47)

[178] Revocacio procuratorum (f. 211)

[179] Renunciacio iuris firme (f. 56)

[180] Rearrendamentum (f. 75)

[181] Renunciacio arrendamenti (f. 79)

[182] Renunciacio ussusfructi racione herencie a marito facta ( $f$. 80)

[183] Ratifficacio et aprobacio sponsalici (f. 82)

[184] Recognicio cuiusdam vendicionis (f. 126)

[185] Recognicio cuiusdam cessionis (f. 208)

[186] Recognicio cuiusdam censualis (f. 109)

[187] Recognicio feci renunciacio cuiusdam censualis (f. 209)

[188] Recognicio cuiusdam arrendamenti (f. 212)

[189] Rearrendamenti domorum (f. 140)

[190] Sindicatus seu procuracionis a quadam universitate (f. 100)

[191] Stabilimentum (f. 38)

[192] Stimacio bonorum cum authoritate et decreto per [...] (f. 184)

[193] Stimacio bonorum mobilium (f. 201)

[194] Substitucio procuratoris (f. 202)

[195] Testamentum seu ultime voluntatis cum eius publicacione (f. 41)

[196] Testamentum cum eius publicacione ( $f$. 41)

[197] Vendicio servi ad usum cosario (f. 23)

[198] Vendicio servi ad usum cosariorum et ad forum Valencie (f. 25)

[199] Vendicio violarii cum mutacione vite in alias vitas (f. 28)

[200] Vendicio equorum (f. 54)

[201] Vendicio prothocollorum et notularum ( $f$. 81)

[202] Vendicio cuiusdam camere prout in prothocollo (f. 156)

[203] Vendicio domorum prout in prothocollo (f. 168)

[204] Vendicio hereditatem prout in prothocollo (f. 170)

[205] Vendicio dot vivi (f. 242)

[206] Vendicio census ad opus solvendi alium censum (f. 154) 
[207] Vendicio terrarum cum magna narrativa facta de verbo et nus redigendo insertis cum firma domini directi (f. 60)

[208] Vendicio domorum prout in prothocollo (f. 235)

[209] Vendicio domorum ad opus licendi et quitandi (f. 252).

FORMULARIO DE ¿ALONSO MUÑOZ?

[1] Instrumentum compromissi (f. 1 r. -4 v.)

[2] Instrumentum cessionis et consignasionis (f. 5 r. -7 r.)

[3] Instrumentum cessioni et iurium renunciacionis (f. $7 \mathrm{r} .-9 \mathrm{v}$.)

[4] Certificatio vite (f. 9 v. -10 r.)

[5] Instrumentum procuracionis coram Sacra Catholica ac Regie Magestate Philipi regis ( $f .10 \mathrm{r} .-12 \mathrm{v}$.)

[6] Instrumentum delegacionis iudicis (f. 12 v.-14 r.)

[7] Instrumentum promisionis et obligationis (f. 14 r.-16 r.)

[8] Instrumentum procuracionis ad lites (f. $16 \mathrm{r} .-\mathrm{v}$.)

[9] Instrumentum procuracionis ad habendum prendam (f. $16 \mathrm{v}$.)

[10] Admissionem ad sepelliendum (f. 17 r.-18 v.)

[11] Instrumentum vendicionis cuiusdam domus (f. $18 \mathrm{v} .-23 \mathrm{v}$.)

[12] Instrumentum afirmacionis ( $f .23$ v. -25 v.)

[13] Instrumentum cessionis (f. 25 v. -27 r.)

[14] Instrumentum indempnitatis (f. $27 \mathrm{v} .-29 \mathrm{v}$.)

[15] Instrumentum exhemptionis (f. $29 \mathrm{v} .-31 \mathrm{r}$.)

[16] Instrumentum magisterii (f. 31 r. -33 v.)

[17] Instrumentum stablimenti (f. 34 r.-39 v.)

[18] Instrumentum procuracionis (f. 39 v. -40 r.)

[19] Instrumentum apoca (f. 40 r.)

[20] Instrumentum relogerii (f. $40 \mathrm{v},-42$ r.)

[21] Instrumentum transactionis (f. 42 r. $-v$.)

[22] Instrumentum consignationis ( $f .42 \mathrm{v}$.)

[23] [Testamento] (f. 43 r. -46 v.)

[24] Vendicion de heredad (f. 49 r. -56 v.)

[25] Consignaçion de mil quinientos sueldos (f. 57 r.-60 r.)

FORMULARIO DE ¿PEDRO PARDO?

[1] Escriptura de articles y capitols de millores (f. $17 \mathrm{v} .-19 \mathrm{r}$.)

[2] Escriptura de variació de eixecució de un tribunal a altre (f. 19 r.-20 r.)

[3] Escriptura de variació de causa de execució de fet (f. 20 r.-21 v.)

[4] Escriptura de deseixida de herènsia faedora per lo hereu molest de deutes del testador (f. 21 v.-22 v.)

[5] Decretació de cura a la menor edad (f. 22 v.-23 v.) 
[6] Decretació de cura al menor e instància de acreedor (f. 23 v.)

[7] Decretació de cura a la absència a instància de acreedor (f. 23 v.-24 v.)

[8] Ampliació de cura a instància de acreedor (f. 24 v.-25 r.)

191 Escriptura de demanda de salaris a instància de procurador o administrador (f. 25 r.-26 r.)

[10] Escriptura de aliments per al menor fahedora per lo curador (f. 26 r. -27 r.)

[11] Escriptura de declaració de major edat (f. 27 r.-v.)

[12] Escriptura de petició de contes de cura o de administració (f. 27 v. -28 r.)

[13] Escriptura de excixida de contes de cura faedora per lo curador e son hereu (f. 28 v.-34 r.)

[14] Declaració de successió ab intestat (f. $34 \mathrm{r}$.-v.)

[15] Declaració de succesió iure vinculi (f. 35 r.-36 r.)

[16] Demanda de reivindicació (f. 36 r.-v.)

[17] Escriptura de capítols (f. 36 v. -39 r.)

[18] Escriptura de interrogatoris (f. 39 r.-v.)

[19] Escriptura de respostes (f. 39 v. -40 r.)

[20] Escriptura de ferma de dret de posesió temporal (f. 40 r.)

[21] Declinatoria de for (f. $40 \mathrm{v} .-41 \mathrm{r}$.)

[22] Escriptura de ferma de dret de posessió imemorial (f. 41 r.-42 r.)

[23] Raons a la ferma de dret (f. 42 v. -43 r.)

[24] Escriptura de nulitats (f. 43 r.-v.)

[25] Apel.lació simple de sentència (f. 44 r.-v.)

[26] Introducció de causa de apelació (f. 44 v.-45 r.)

[27] Escriptura de mandas de comís per lo señor directe (f. 46 r.-v.)

[28] A de comparendo per demanar revisió de alguna provisió o manament en tribunals inferior eo correcçió (f. 46 v. -47 r.)

[29] Acte de comparendo per a renunsiar a la instànsia de ferma de dret (f. 47 r.)

[30] Yntima extrajudicial faedora per lo comprador a rahó de franch a qui li demanen sert sens sobre la cosa comprada (f. 47 r. $-49 \mathrm{v}$.)

[31] Supplicació per a quant una dona se ha fet pagament y vol que no la molesten los acreedors del marit en los bens del pagament (f. 49 v. -50 r.)

[32] Decret per a que un curador puga fermar en la venda de una cosa en la qual juntament $a b$ lo menor y altres señors pro indiviso y estos no volen estar en comunió (f. 50 r.-51 r.)

[33] Recors de señoría directa per a quant a hu el citen a cabrebar y no consta de la dita señoría (f. 51 r. -52 v.)

[34] Petició de evocació de ferma de dret (f. 52 r.-v.)

[35] Escriptura de recors de alguns injusts enantaments (f. 52 v. $-53 v$ v.)

[36] Suplicació per a quant dos causes que sendunt ad eundem estan en dos oidors es lleve la comisió al hu y se cometa a l'altre (f. 53 v. -54 r.)

[37] Operis nove mutatione en nom de hereu (f. 54 r.-v.)

[38] Petíció per a que es reba informació per a probar que una persona de edat de 17 anys per a efecte que sa magestat faça gràsia de suplement de edat (f. 55 r.- v.)

[39] Petició de oposición para el obtento de algun beneficio jure propio (f. 55 v. -56 v.) 
[40] Scriptura per a probar una lletra si es de la mà de alguna persona (f. 56 v. -57 r.)

[41] Escriptura per a fer deposit del preu de la cosa venuda moble per cort faedora per lo comprador (f. 57 v.-58 r.)

[42] Escriptura de declaració de miserables la qual se ha de posar per lo tribunal y trast de aquell a carrech de qui està presa la miserable persona (f. 58 r. -59 r.)

[43] Scriptura per a demanar una terra o cosa comprada en virtut de la fadiga (f. 59 r. $-60 \mathrm{v}$.)

[44] Denunciació per rahó de estupro fahedora per lo pare o mare de la fadrina (f. 60 v. $-63 \mathrm{r}$.)

[45] Petició per a quant una fadrina ha demanat a algú ratione stupri demanar visura ut constet de corpore delicti (f. 63 r.-v.)

[46] Demanda de divorcio que se ha de poner por la muger molestada de su marido ante el vicario general (f. 63 v. -65 r.)

[47] Escriptura de respostes per a que responguen los de una comunitat y universitat cada hu de per sí (f. 65 v. -66 r.)

[48] Decret per a vendre bens de un menor (f. 66 r. $-67 \mathrm{v}$.)

[49] Memorial per a nombrar-se segrestador (f. 67 v.-68 r.)

[50] Dissentiment (f. 67 v.-68 r.)

[51] Suplicació de recors en cas de negar-se la supplicació de una sentència eo provisió per a la altra sala (f. $68 \mathrm{r}$.-v.)

[52] Supplicació de introducció de fet en la altra real sala denegada la supplicació y recors (f. 69 r.-v.)

[53] Supplicació per a traure ab fianses a un malalt de la presó (f. 69 v. -70 v.)

[54] Tramesa de cort pro luitione y per a buscar papers (f. $70 \mathrm{v} .-71 \mathrm{v}$.)

[55] Variació de un tribunal a altre (f. 72 r.-v.)

[57] Demanda de tenuta (f. 72 v. -73 r.)

[58] Demanda de restitució de dot (f. 73 r.-74 r.)

[59] Recors (f. 74 v. -75 v.)

[60] Cumulació (f. 76 r.-v.)

[61] Escriptura per a que hu que ha venut unes terres de un vincle fasa fermar al succesor en aquell o que restitueixca lo preu de dites terres estant prompte lo comprador a tornar-li aquelles (f. 77 r.-v.)

[62] Denunciació criminal en proçés de ausència (f. 77 v.-78 v.)

[63] Denunciació en procés de presència (f. 78 v. -80 r.)

[64] Supplicació de avehinament (f. 80 r.-81 v.)

[65] Demanda de aliments feta per lo herma menor al major (f. 81 v. -82 v.)

[66] Supplicació per a ser admés a donar hu dita en paga de son deute ex quo no y .a concurs de acreedors (f. 82 v. -83 r.)

[67] Condemnació de dot (f. 83 r.-84 v.)

[68] Escriptura de avehinament ( $f .84$ v. -85 v.)

[69] Escriptura per a que es declare anulant una institució de herènsia per haver deixat la mare més al padastre que a una filla de primer matrimoni (f. 85 v. -86 v.)

[70] Escriptura de interrogatoris a una escriptura de capítols en que es vol probar la lletra de la ferma de una persona (f. 86 v.-97 v.)

[71] Escriptura per a declarar-se haver succehit un combent en los bens de una monga per no tenir fet testament ( $f .87 \mathrm{v} .-88 \mathrm{v}$.) 